\title{
Imaginary-Time Path Integral for a Relativistic Spinless Particle in an Electromagnetic Field
}

\author{
Takashi Ichinose ${ }^{1}$ and Hiroshi Tamura ${ }^{2}$ \\ 1 Department of Mathematics, Kanazawa University, 920 Kanazawa, Japan \\ 2 Department of Physics, Hokkaido University, 060 Sapporo, Japan
}

\begin{abstract}
A rigorous path integral representation of the solution of the Cauchy problem for the pure-imaginary-time Schrödinger equation $\partial_{t} \psi(t, x)=$ $-\left[H-m c^{2}\right] \psi(t, x)$ is established. $H$ is the quantum Hamiltonian associated, via the Weyl correspondence, with the classical Hamiltonian $\left[(c p-e A(x))^{2}+\right.$ $\left.m^{2} c^{4}\right]^{1 / 2}+e \Phi(x)$ of a relativistic spinless particle in an electromagnetic field. The problem is connected with a time homogeneous Lévy process.
\end{abstract}

\section{Introduction}

The Feynman-Kac-Itô formula is an imaginary-time path integral for a nonrelativistic spinless particle in an electromagnetic field representing the solution of the Cauchy problem for the pure-imaginary-time Schrödinger equation, i.e. the heat equation (e.g. Simon [27]).

As concerns a relativistic particle, Ichinose-Tamura [13] (cf. $[14,15]$ ) have established a path integral formula for the Dirac particle in $1+1$-dimensional space-time to represent the solution of the Dirac equation. There is constructed a matrix-valued measure on the space of the Lipschitz-continuous paths. Relevant problems are also discussed by Gaveau et al. [9 and 8] with Poisson processes.

The aim of the present paper is to give an imaginary-time path integral for a relativistic spinless particle of mass $m>0$ and charge $e$ interacting with an electromagnetic field which represents the solution $\psi(t, x)$ of the Cauchy problem for the pure-imaginary-time Schrödinger equation,

$$
\partial_{t} \psi(t, x)=-\left[H-m c^{2}\right] \psi(t, x), \quad t>0, \quad x \in \mathbb{R}^{d},
$$

the dimension $d$ of space $\mathbb{R}^{d}$ being arbitrary. Here $H$ is the quantum Hamiltonian associated, via the Weyl correspondence, with the classical Hamiltonian

$$
\begin{aligned}
h(p, x) & =h_{A}(p, x)+e \Phi(x), \\
h_{A}(p, x) & =\left[(c p-e A(x))^{2}+m^{2} c^{4}\right]^{1 / 2}, \quad p \in \mathbb{R}^{d}, \quad x \in \mathbb{R}^{d},
\end{aligned}
$$

for the particle. $A(x)$ and $\Phi(x)$ are respectively the vector and scalar potentials of the 
field (e.g. Landau-Lifschitz [20]). $c$ is the light velocity. This Hamiltonian may be of some interest in the region where relativity is of some importance but where quantum field theoretic effects are not yet important (See [27, p. 221]).

We assume for simplicity that $A$ is in $\mathscr{B}\left(\mathbb{R}^{d} \rightarrow \mathbb{R}^{d}\right)$ and $\Phi$ in $\mathscr{B}\left(\mathbb{R}^{2} \rightarrow \mathbb{R}\right)$, where $\mathscr{B}\left(\mathbb{R}^{d} \rightarrow \mathbb{R}^{N}\right), N=1, d$, is the Fréchet space of the $\mathbb{R}^{N}$-valued $C^{\infty}$ functions in $\mathbb{R}^{d}$ which together with their derivatives of all orders are bounded. The definition of $H$ is given by

$$
\begin{aligned}
H g & =\left[H_{A}+e \Phi\right] g, \\
\left(H_{A} g\right)(x) & =(2 \pi)^{-d} \iint_{\mathbb{R}^{d} \times \mathbb{R}^{d}} e^{i(x-y) \cdot p} h_{A}\left(p, \frac{x+y}{2}\right) g(y) d y d p
\end{aligned}
$$

with (1.2) for $g \in \mathscr{S}\left(\mathbb{R}^{d}\right)$ (e.g. $\left.[1,10,12]\right)$. The Planck constant $\hbar$ is taken to equal 1. The integral in (1.3) is an oscillatory integral (e.g. Kumano-go [19]). It is seen that $H$ maps $\mathscr{S}\left(\mathbb{R}^{d}\right)$ into itself, so that it defines a linear operator in $L^{2}\left(\mathbb{R}^{d}\right)$. It is shown that $H$ is essentially selfadjoint on $C_{0}^{\infty}\left(\mathbb{R}^{d}\right)$, and its closure, denoted also by the same $H$, is bounded from below. It is this selfadjoint operator $H$ that is in (1.1). Notice that $H_{A}$ differs from the square root of the nonnegative selfadjoint operator $(-c i \partial-$ $e A(x))^{2}+m^{2} c^{4}$.

Our approach is a rigorous application of the phase space path integral or Hamiltonian path integral method with the "midpoint" prescription (Mizrahi $[21,22]$, Garrod [7]). The path space measure used is the probability measure $\lambda_{0, x}$ on the space of the right-continuous paths $X:[0, \infty) \rightarrow \mathbb{R}^{d}$ having the left-hand limits and satisfying $X(0)=x$ whose characteristic function is

$$
\exp \left\{-t\left[\left(c^{2} p^{2}+m^{2} c^{4}\right)^{1 / 2}-m c^{2}\right]\right\}=\int e^{i p \cdot(X(t)-X(0))} d \lambda_{0, x}(X) .
$$

The path $X$ is called a d-dimensional time homogeneous Lévy process (e.g. IkedaWatanabe [16], Itô [17]). The formula obtained shows a close analogy with the Feynman-Kac-Itô formula based on the Wiener measure. Similar treatments will be possible for $t$-dependent vector and scalar potentials $A(t, x)$ and $\Phi(t, x)$.

In Sect. 2 our result is stated. Sections 3 and 4 are devoted to its proof. Section 5 is concerned with an extension to the case of nonregular and unbounded vector and scalar potentials. In Sect. 6 our approach is heuristically examined with the phase space path integral or Hamiltonian path integral method.

In a subsequent paper the nonrelativistic limit problem will be discussed.

\section{Path Integral Formula}

The main result, summarized in the theorem below, is a path integral representation of the solution $\psi(t, x)$ of the Cauchy problem for (1.1) with initial data $\psi(0, x)=g(x)$. Its statement needs some notions on the Lévy process, which we shall explain after the theorem. In Sect. 5 we indicate the extension to the case of nonregular and unbounded vector and scalar potentials.

Theorem 2.1. Assume that $A$ is in $\mathscr{B}\left(\mathbb{R}^{d} \rightarrow \mathbb{R}^{d}\right)$ and $\Phi$ in $\mathscr{B}\left(\mathbb{R}^{d} \rightarrow \mathbb{R}\right)$. Then:

(i) Both $H_{A}$ and $H$, defined by (1.3), are essentially selfadjoint on $C_{0}^{\infty}\left(\mathbb{R}^{d}\right)$. Their closures, denoted by the same $H_{A}$ and $H$, are bounded from below and have domain $H^{1}\left(\mathbb{R}^{d}\right)$. 
(ii) There exists a countably additive probability measure $\lambda_{0, x}$ on the path space $D_{0, x}\left([0, \infty) \rightarrow \mathbb{R}^{d}\right)$ of the right-continuous paths $X:[0, \infty) \rightarrow \mathbb{R}^{d}$ having the left-hand limits and satisfying $X(0)=x$ such that whenever $A$ is in $\mathscr{B}\left(\mathbb{R}^{d} \rightarrow \mathbb{R}^{d}\right)$ and $\Phi$ in $\mathscr{B}\left(\mathbb{R}^{d} \rightarrow \mathbb{R}\right)$, it holds that

$$
\psi(t, x)=\left(e^{-t\left[H-m c^{2}\right]} g\right)(x)=\int e^{-S(t, 0 ; X)} g(X(t)) d \lambda_{0, x}(X)
$$

for $g \in L^{2}\left(\mathbb{R}^{d}\right)$. Here

$$
\begin{aligned}
S(t, 0 ; X)= & i \int_{0}^{t+} \int_{|y| \geqq 1}(e / c) A(X(s-)+y / 2) \cdot y N_{X}(d s d y) \\
& +i \int_{0}^{t+} \int_{0<|y|<1}(e / c) A(X(s-)+y / 2) \cdot y \tilde{N}_{X}(d s d y) \\
& +i \int_{0}^{t} \int_{0<|y|<1}(e / c)[A(X(s)+y / 2)-A(X(s))] \cdot y \hat{N}(d s d y)+\int_{0}^{t} e \Phi(X(s)) d s \\
= & i \int_{0}^{t+} \int_{|y|>0}(e / c) A(X(s-)+y / 2) \cdot y \tilde{N}_{X}(d s d y) \\
& +i \int_{0|y|>0}^{t} \int_{|c| c \mid c}(e / c)[A(X(s)+y / 2)-A(X(s))] \cdot y \hat{N}(d s d y)+\int_{0}^{t} e \Phi(X(s)) d s .
\end{aligned}
$$

(iii) Consequently, $H_{A}-m c^{2}$ is nonnegative selfadjoint.

Each of those paths $X$ on which the measure $\lambda_{0, x}$ is concentrated is called a $d$ dimensional time homegeneous Lévy process which does not contain the continuous martingale part. The Lévy-Itô theorem states, in fact,

$$
\begin{aligned}
X(t) & =X(0)+\int_{0}^{t+} \int_{|y| \geq 1} y N_{X}(d s d y)+\int_{0}^{t+} \int_{0<|y|<1} y \tilde{N}_{X}(d s d y) \\
& =X(0)+\int_{0}^{t+} \int_{|y|>0} y \tilde{N}_{X}(d s d y) .
\end{aligned}
$$

With $\mathbb{D}_{X} \equiv\{s>0 ; X(s) \neq X(s-)\}$, the jump $X(s)-X(s-), s \in \mathbb{D}_{X}$, is a stationary Poisson point process on $\mathbb{R}^{d} \backslash\{0\} . N_{X}(d s d y)$ is a counting measure on $(0, \infty) \times$ $\left(\mathbb{R}^{d} \backslash\{0\}\right)$ :

$$
N_{X}\left(\left(t, t^{\prime}\right] \times U\right)=\#\left\{\mathrm{~s} \in\left(t, t^{\prime}\right] ; \quad X(s) \neq X(s-), \quad X(s)-X(s-) \in U\right\},
$$

where $0<t<t^{\prime}$ and $U$ is a Borel subset of $\mathbb{R}^{d} \backslash\{0\} . \tilde{N}_{X}(d s d y)$ is by definition

$$
\tilde{N}_{X}(d s d y)=N_{X}(d s d y)-\hat{N}(d s d y)
$$

where

$$
\hat{N}(d s d y) \equiv \int N_{X}(d s d y) d \lambda_{0, x}(X)=d s n(d y)
$$

is called the compensator of $N_{X}(d s d y)$, and $n(d y)$ is the Lévy measure. The LévyKhinchin formula connects $\lambda_{0, x}$ with $n(d y)$ as follows

$$
e^{-(t-s) a(p)}=\int e^{i p \cdot(X(t)-X(s))} d \lambda_{0, x}(X), \quad 0 \leqq s \leqq t, \quad p \in \mathbb{R}^{d},
$$

where

$$
a(p) \equiv\left(c^{2} p^{2}+m^{2} c^{4}\right)^{1 / 2}-m c^{2}=-\int_{\mathbb{R}^{d} \backslash\{0\}}\left[e^{i p \cdot y}-1-i p \cdot y I_{\{|y|<1\}}(y)\right] n(d y)
$$


with $I_{\{|y|<1\}}(y)=1$ if $|y|<1$, and $=0$ if $|y| \geqq 1$. It is known that the Lévy measure $n(d y)$ is a $\sigma$-finite measure on $\mathbb{R}^{d} \backslash\{0\}$ such that

$$
\int_{\mathbb{P}^{d} \backslash\{0\}}\left[y^{2} /\left(1+y^{2}\right)\right] \mathrm{n}(d y)<\infty .
$$

Further we see in our case, since $a(p)$ is analytic in $p$, applying to both sides of (2.5) $-\Delta_{p}$, the minus Laplacian in the variable $p$, to the $k$ th power, $k \geqq 1$, that $n(d y)$ satisfies

$$
\int_{\mathbb{R}^{d} \backslash\{0\}} e^{i p \cdot y}|y|^{2 k} n(d y)=-\left(-\Delta_{p}\right)^{k}\left(c^{2} p^{2}+m^{2} c^{4}\right)^{1 / 2}
$$

if $m c>0$, and in particular,

$$
\int_{\mathbb{R}^{d} \backslash\{0\}}|y|^{2 k} n(d y)<\infty .
$$

We note that the second equality in (2.2) as well as (2.3) is due to the fact that $\int y n(d y)=0$ because of the rotational symmetry of $n(d y)$.

${ }^{|y| \geqq 1}$ For the Lévy process we refer to Ikeda-Watanabe [16] and Itô [17]. Finally we give a notational comment. In Ikeda-Watanabe [16] the Poisson point process is denoted by $p(s)=X(s)-X(s-), s \in \mathbb{D}_{X}$, and our $N_{X}, \tilde{N}_{X}$ and $\hat{N}$ by $N_{p}, \tilde{N}_{p}$ and $\hat{N}_{p}$.

\section{Proof of Theorem 2.1(i)}

The following theorem is more precise than Theorem 2.1(i).

Theorem 3.1. (i) There exists a constant $C>0$ such that

$$
\left\|H_{A} g\right\|_{0} \leqq C\|g\|_{1}, \quad g \in S\left(\mathbb{R}^{d}\right) .
$$

(ii) (Gårding's inequality) There exists a constant $C(m, c)>0$ possibly depending on $m>0$ and $c>0$ such that

$$
\left(H_{A} g, g\right) \geqq m c^{2}\left\|\left[-\left(1 / m^{2} c^{2}\right) \Delta+1\right]^{1 / 4} g\right\|_{0}^{2}-C(m, c)\|g\|_{0}^{2},
$$

for $g \in \mathscr{S}\left(\mathbb{R}^{d}\right) . C(m, c)$ is a constant independent of $m$ and $c$ if $m c \geqq 1$ and $c \geqq 1$.

(iii) Both $H_{A}$ and $H$, as operators in $L^{2}\left(\mathbb{R}^{d}\right)$ with domain $C_{0}^{\infty}\left(\mathbb{R}^{d}\right)$, are essentially selfadjoint. Here $\|\cdot\|_{s}$ stands for the norm of the Sobolev space $H^{s}\left(\mathbb{R}^{d}\right)$.

Proof. (i) Since $A$ is $C^{\infty}$ and bounded, and all its derivatives are bounded, the symbol $h_{A}$ in (1.2) satisfies that for all multi-indices $\alpha, \beta$ there is a constant $C_{\alpha \beta}$ such that

$$
\left|\partial_{p}^{\alpha} \partial_{x}^{\beta} h_{A}(p, x)\right| \leqq C_{\alpha \beta}\left(1+p^{2}\right)^{(1-|\alpha|) / 2}, \quad(p, x) \in \mathbb{R}^{d} \times \mathbb{R}^{d} .
$$

Then (3.1) is an immediate consequence of the Calderón-Vaillancourt theorem $([2,18])$.

(ii) We have

$$
h_{A}\left(p, \frac{x+y}{2}\right)=h_{0}(p)+r(x, p, y)
$$


with

$$
h_{0}(p)=\left(c^{2} p^{2}+m^{2} c^{4}\right)^{1 / 2}
$$

and

$$
r(x, p, y)=-e A\left(\frac{x+y}{2}\right) \cdot\left(2 c p-e A\left(\frac{x+y}{2}\right)\right) /\left[h_{0}(p)+h_{A}\left(p, \frac{x+y}{2}\right)\right] .
$$

It follows that $h_{0}(p) \geqq m c^{2}$, and for all multi-indices $\alpha, \beta$ and $\gamma$, there is a constant $C_{\alpha \beta \gamma}>0$ independent of $m$ and $c$ where $m c \geqq 1$ and $c \geqq 1$ such that

$$
\left|\partial_{p}^{\alpha} \partial_{x}^{\beta} \partial_{y}^{\gamma} r(x, p, y)\right| \leqq C_{\alpha \beta \gamma}\left(p^{2}+m^{2} c^{2}\right)^{-|\alpha| / 2}, \quad p \in \mathbb{R}^{d},(x, y) \in \mathbb{R}^{d} \times \mathbb{R}^{d}
$$

We have $H_{A}=H_{0}+R$, where

$$
H_{0}=\left(-c^{2} \Delta+m^{2} c^{4}\right)^{1 / 2},
$$

and

$$
(R g)(x)=(2 \pi)^{-d} \iint_{\mathbb{R}^{d} \times \mathbb{R}^{d}} e^{i(x-y) \cdot p} r(x, p, y) g(y) d y d p, \quad g \in \mathscr{S}\left(\mathbb{R}^{d}\right) .
$$

It is clear that for $g \in \mathscr{S}\left(\mathbb{R}^{d}\right)$,

$$
\left(H_{0} g, g\right)=m c^{2}\left\|\left[-\left(1 / m^{2} c^{2}\right) \Delta+1\right]^{1 / 4} g\right\|_{0}^{2},
$$

and the Calderón-Vaillancourt theorem $([2,18])$ yields

$$
\|R g\|_{0} \leqq C(m, c)\|g\|_{0}, \quad g \in \mathscr{S}\left(\mathbb{R}^{d}\right),
$$

where

$$
C(m, c)=C \max _{|\alpha| \leqq 2([d / 2]+1)} \max _{p}\left[\left(p^{2}+1\right) /\left(p^{2}+m^{2} c^{2}\right)\right]^{|\alpha| / 2}
$$

with a constant $C$ which is independent of $m$ and $c$ if $m c \geqq 1$ and $c \geqq 1$, so that $C(m, c) \leqq C$ if $m c \geqq 1$ and $c \geqq 1$. For $r \geqq 0,[r]$ denotes the largest integer that satisfies $[r] \leqq r$.

(iii) We follow the argument of Shubin [26]. The pseudo-differential operator $H$ is formally selfadjoint. It maps $\mathscr{S}\left(\mathbb{R}^{d}\right)$ into itself, and $\mathscr{S}^{\prime}\left(\mathbb{R}^{d}\right)$ into itself. Denote by $T$ the corresponding operator in $L^{2}\left(\mathbb{R}^{d}\right)$ with domain $D[T]=C_{0}^{\infty}\left(\mathbb{R}^{d}\right) . T$ is symmetric. So $T$ is essentially selfadjoint if and only if its deficiency indices $\left(n_{+}, n_{-}\right)$vanish. To show $n_{+}=0$, let $u \in L^{2}\left(\mathbb{R}^{d}\right)$ be orthogonal to the range of $T+i$. If $T^{*}$ is the Hilbert space adjoint of $T$, it follows that $u \in D\left[T^{*}\right]$ and $\left(T^{*}-i\right) u=0$ or $(H-i) u=0$. Since $H-i$ is elliptic as seen from $(1.2)$, we have $u \in H^{\infty}\left(\mathbb{R}^{d}\right)=\bigcap_{s} H^{s}\left(\mathbb{R}^{d}\right)$. Then we claim that

$$
\left(T^{*} u, u\right)=\left(u, T^{*} u\right)
$$

To show this, choose a sequence $\left\{u_{n}\right\}$ in $C_{0}^{\infty}\left(\mathbb{R}^{d}\right)$ which converges to $u$ in $H^{1}$. Then

$$
\left(T^{*} u, u\right)=\lim \left(T^{*} u, u_{n}\right)=\lim \left(u, T u_{n}\right) .
$$

The inequality (3.1) implies that $T u_{n}=H u_{n}$ is convergent to an element $v$ in $L^{2}\left(\mathbb{R}^{d}\right)$. Since $T$ is closable with closure $T^{*}$, we have $v=T^{*} u$. This proves our claim. From 
(3.5) we conclude $u=0$, i.e. $n_{+}=0$, because

$$
-i(u, u)=\left(T^{*} u, u\right)=\left(u, T^{*} u\right)=i(u, u) .
$$

Similarly we can prove $n_{-}=0$, completing the proof of Theorem 3.1 .

\section{Proof of Theorem 2.1 (ii) and (iii)}

We mimic the proof of the Feynman-Kac-Itô formula in [27]. The first three subsections concern the proof of Theorem 2.1 (ii). First we describe what is our path space measure $\lambda_{0, x}$. Next we construct the semigroup solution of the Cauchy problem for (1.1). Finally we establish the path integral representation (2.1) with (2.2). In the last subsection the statement (iii) is proved.

4.1. The Path Space Measure $\lambda_{0, x}$. Consider the Cauchy problem for the free equation

$$
\begin{aligned}
\partial_{t} \psi(t, x) & =-\left[H_{0}-m c^{2}\right] \psi(t, x) \\
& \equiv-\left[\left(-c^{2} \Delta+m^{2} c^{4}\right)^{1 / 2}-m c^{2}\right] \psi(t, x) \quad t>0, x \in \mathbb{R}^{d},
\end{aligned}
$$

to (1.1) with initial data $\psi(0, x)=g(x)$.

Lemma 4.1. (i) For each $t>0$, the function $e^{-t a(p)}$ with $a(p)$ in (2.5) is of positive type in $p \in \mathbb{R}^{d}$, and the Lévy-Khinchin formula (2.5) holds.

(ii) If $k_{0}(t, x)$ is the fundamental solution of the Cauchy problem for (4.1), then for $m>0$,

$$
\begin{aligned}
k_{0}(t, x)= & 2^{-(d-1) / 2} \pi^{-(d+1) / 2} m^{(d+1) / 2} c^{(d+3) / 2} e^{m c^{2} t} \\
& \times t\left(x^{2}+c^{2} t^{2}\right)^{-(d+1) / 4} K_{(d+1) / 2}\left(m c\left(x^{2}+c^{2} t^{2}\right)^{1 / 2}\right),
\end{aligned}
$$

where $K_{v}(\tau)$ is the modified Bessel function of the third kind with order $v$. In particular, $k_{0}(t, x)$ is positive and

$$
\int_{\mathbb{R}^{d}} k_{0}(t, x) d x=1
$$

Proof. (i) $a(p)$ is spherically symmetric in $p$ with $a(p) \geqq a(0)=0$, and

$$
\Delta a(p)=2 c^{2}\left(c^{2} p^{2}+m^{2} c^{4}\right)^{-1 / 2}+m^{2} c^{6}\left(c^{2} p^{2}+m^{2} c^{4}\right)^{-3 / 2}
$$

is a function of positive type, since, for each $t>0, \exp \left[-t c^{2} p^{2}\right]$ is a function of positive type, and so is the function

$$
\left(c^{2} p^{2}+m^{2} c^{4}\right)^{-\beta}=d_{\beta} \int_{0}^{\infty} t^{\beta-1} \exp \left[-t\left(c^{2} p^{2}+m^{2} c^{4}\right)\right] d t
$$

with $\beta>0$ and a positive constant $d_{\beta}$ depending on $\beta$. It follows by [27, Theorem XIII. 54, p. 219] that $a(p)$ is conditionally negative definite, and further by [27, Theorem XIII. 52, p. 214] that for each $t>0, e^{-t a(p)}$ is a function of positive type.

(ii) First we put $c=1$. Using the spherical coordinates and an integral representation of the Bessel function $J_{v}$ [5, Chap. VII, 7.3.2. (3), p. 14] we obtain 


$$
\begin{aligned}
k_{0}(t, x) & =(2 \pi)^{-d} \int_{\mathbb{R}^{d}} e^{i p \cdot x} e^{-t a(p)} d p \\
& =c_{d} e^{m t} \int_{0}^{\infty} \exp \left[-t\left(\rho^{2}+m^{2}\right)^{1 / 2}\right] \rho^{d-1} d \rho \int_{0}^{\pi} \cos (r \rho \cos \theta)(\sin \theta)^{d-2} \mathrm{~d} \theta \\
& =(2 \pi)^{-d / 2} e^{m t} r^{-(d-1) / 2} \int_{0}^{\infty} \rho^{(d-1) / 2} \exp \left[-t\left(\rho^{2}+m^{2}\right)^{1 / 2}\right](r \rho)^{1 / 2} J_{(d-2) / 2}(r \rho) d \rho
\end{aligned}
$$

with $c_{d}=2^{1-d} \pi^{-(d+1) / 2} \Gamma((d-1) / 2)^{-1}$. Calculation of the integral in the last member, i.e. the Hankel transform (use [6, Chap. VIII, 8.6. (19), p. 31, where read $-v / 2-3 / 4$ instead of $-v-3 / 4]$ ) yields $(4.2)$ with $c=1$. For general $c>0$ replace $t$ by $c t$ and $m$ by $m c$. This ends the proof of Lemma 4.1 .

In connection with Lemma 4.1 , it is known that for each $x \in \mathbb{R}^{d}$ there exists a countably additive measure $\lambda_{0, x}$ on the space $D_{0, x}\left([0, \infty) \rightarrow \mathbb{R}^{d}\right)$ of the rightcontinuous paths $X(s)$ having the left-hand limits and satisfying $X(0)=x$ such that if $\varphi(X)=F\left(X\left(t_{0}\right), \ldots, X\left(t_{n}\right)\right)$ with $0=t_{0}<t_{1}<\cdots<t_{n}=t$ and with $F\left(x^{(0)}, \ldots, x^{(n)}\right)$ a bounded continuous function on $\mathbb{R}^{d(n+1)}$, then

$$
\begin{aligned}
\int \varphi(X) d \lambda_{0, x}(X)= & \overbrace{\mathbb{R}^{d}}^{n} \cdots \int_{\mathbb{R}^{d}}^{n} k_{0}\left(t_{1}-t_{0}, x^{(0)}-x^{(1)}\right) \cdots \cdot k_{0}\left(t_{n}-t_{n-1}, x^{(n-1)}-x^{(n)}\right) \\
& \cdot F\left(x^{(0)}, \ldots, x^{(n)}\right) d x^{(1)} \ldots \cdot d x^{(n)}
\end{aligned}
$$

with $x^{(0)}=x$. The path $X(s)$ is a $d$-dimensional time homogeneous Lévy process. We have (2.3), (2.4) and (2.5) (See [16,17]).

4.2. Semigroup Solution of the Cauchy Problem. We begin with a lemma about the operator $T(\tau), \tau>0$, defined by

$$
(T(\tau) g)(x)=\int_{\mathbb{R}^{d}} k_{0}(\tau, x-y) \exp \left[i(e / c) A\left(\frac{x+y}{2}\right) \cdot(x-y)-e \Phi\left(\frac{x+y}{2}\right) \tau\right] g(y) d y
$$

for $g \in L^{2}\left(\mathbb{R}^{d}\right)$.

Lemma 4.2. If $A$ is in $\mathscr{B}\left(\mathbb{R}^{d} \rightarrow \mathbb{R}^{d}\right)$ and $\Phi$ in $\mathscr{B}\left(\mathbb{R}^{d} \rightarrow \mathbb{R}\right)$, then $T(\tau)$ defines a bounded linear operator of $L^{2}\left(\mathbb{R}^{d}\right)$ into itself and $\|T(\tau)\| \leqq e^{M \tau}$ with $M=\left(-\inf _{x \in \mathbb{R}^{d}} e \Phi(x)\right) \vee 0$. Further if $g$ is in $D[H]=H^{1}\left(\mathbb{R}^{d}\right)$, then $\partial_{\tau}(T(\tau) g)$ approaches $-\left[H-m c^{2}\right] g$ in $L^{2}$ as $\tau \downarrow 0$.

The proof of Lemma 4.2, which is rather lengthy, is postponed till the end of this subsection.

Next we consider the $n$-times iteration of $T(t / n)$ :

with

$$
\begin{aligned}
\left(T(t / n)^{n} g\right)(x)= & \int_{\mathbb{R}^{d}}^{n} \cdots \int_{\mathbb{R}^{d}}^{n} k_{0}\left(t / n, x^{(0)}-x^{(1)}\right) \cdot \cdots k_{0}\left(t / n, x^{(n-1)}-x^{(n)}\right) \\
& \times \exp \left[-S_{n}\left(x^{(0)}, \ldots, x^{(n)}\right)\right] g\left(x^{(n)}\right) d x^{(1)} \cdots \cdot d x^{(n)}
\end{aligned}
$$

$$
\begin{aligned}
S_{n}\left(x^{(0)}, \ldots, x^{(n)}\right)= & i \sum_{j=1}^{n}(e / c) A\left(\frac{x^{(j-1)}+x^{(j)}}{2}\right) \cdot\left(x^{(j)}-x^{(j-1)}\right) \\
& +\sum_{j=1}^{n} e \Phi\left(\frac{x^{(j-1)}+x^{(j)}}{2}\right)(t / n),
\end{aligned}
$$


for $g \in L^{2}\left(\mathbb{R}^{d}\right)$, where $t>0$ and $x^{(0)}=x$.

In Sect. 4.3 we shall see what the right-hand side of (4.6) approaches as $n \rightarrow \infty$. In the following we show that the left-hand side of (4.6) approaches in $L^{2}$ the solution $\left(e^{-t\left[H-m c^{2}\right]} g\right)(x)$ of the Cauchy problem for (1.1) as $n \rightarrow \infty$ uniformly on compact sets in $t \geqq 0$, and hence it does for a.e. $x$ in $\mathbb{R}^{d}$, by taking a subsequence if necessary.

Since $D[H]$ is dense and the operators $T(t / n)^{n}$ are, by Lemma 4.2, uniformly bounded by $e^{M t}$, we have only to show this for $g \in D[H]$. So let $g \in D[H]$. By Lemma 4.2 we have with $K=H-m c^{2}$,

$$
\begin{aligned}
\left\|T(t / n)^{n} g-e^{-t K} g\right\| & =\left\|\sum_{j=1}^{n} T(t / n)^{j-1}\left(T(t / n)-e^{-(t / n) K}\right) e^{-(n-j)(t / n) K} g\right\| \\
& \leqq n e^{M t} \sup _{0 \leqq s \leqq t}\left\|\left(T(t / n)-e^{-(t / n) K}\right) e^{-s K} g\right\|,
\end{aligned}
$$

which we now show tends to zero uniformly on compact sets in $t \geqq 0$ as $n \rightarrow \infty$. To do so, note that since $H$ is closed, $D \equiv D[H]$ is a Hilbert space equipped with the graph norm of $H$. By Lemma 4.2, $\left\{n\left(T(t / n)-e^{-(t / n) K}\right)\right\}_{n=1}^{\infty}$ is a sequence of bounded operators of the Hilbert space $D$ into $L^{2}\left(\mathbb{R}^{d}\right)$. We have for each fixed $f \in D$,

$$
\begin{aligned}
\left\|n\left(T(t / n)-e^{-(t / n) K}\right) f\right\| & =n\left\|\int_{0}^{t / n} \partial_{\tau}\left(T(\tau)-e^{-\tau K}\right) f d \tau\right\| \\
& \leqq t \sup _{0 \leqq \tau \leqq t / n}\left\|\partial_{\tau}\left(T(\tau)-e^{-\tau K}\right) f\right\| .
\end{aligned}
$$

Then by Lemma 4.2 again we see the last member of (4.8) tends to zero uniformly on compact sets in $t \geqq 0$ as $n \rightarrow \infty$. By the uniform boundedness principle the sequence $\left\{n\left(T(t / n)-e^{-(t / n) K}\right)\right\}$ is, as bounded operators of the Hilbert space $D$ into $L^{2}\left(\mathbb{R}^{d}\right)$, uniformly bounded for all $n$ and on every fixed compact set in $t \geqq 0$. Consequently, it converges to zero uniformly on compact subsets of the Hilbert space $D$. The map $[0, t] \in S \mapsto e^{-s K} g \in D$ is continuous, and so $\left\{e^{-s K} g ; 0 \leqq s \leqq t\right\}$ is a compact subset of the space $D$. This proves the convergence of the left-hand side of (4.6) to the solution of the Cauchy problem for (1.1).

Finally, we prove Lemma 4.2 .

Proof of Lemma 4.2. To avoid unnecessary complexity we put $c=e=1$. The first assertion is easy because the integral operator defined with positive kernel $k_{0}(t, x)$ is a contraction. So we come to the second.

Since $k_{0}(\tau, x)$ is the fundamental solution of the Cauchy problem for (4.1), we have by integration by parts

$$
\begin{aligned}
\left(\partial_{\tau} T(\tau) g\right)(x)= & -\int k_{0}(\tau, x-y)\left[\left(-\Delta_{y}+m^{2}\right)^{1 / 2}-m+\Phi\left(\frac{x+y}{2}\right)\right] \\
& \times\left(\exp \left[i A\left(\frac{x+y}{2}\right) \cdot(x-y)-\Phi\left(\frac{x+y}{2}\right) \tau\right] g(y)\right) d y \\
\equiv & \left(I_{1}(\tau) g\right)(x)+\left(I_{2}(\tau) g\right)(x) .
\end{aligned}
$$


Here $\Delta_{y}$ is the Laplacian in the variable $y$, and

$$
\begin{aligned}
\left(I_{1}(\tau) g\right)(x)= & -\int k_{0}(\tau, x-y)\left(-\Delta_{y}+m^{2}\right)^{1 / 2} \\
& \times\left(\exp \left[i A\left(\frac{x+y}{2}\right) \cdot(x-y)-\Phi\left(\frac{x+y}{2}\right) \tau\right] g(y)\right) d y, \\
\left(I_{2}(\tau) g\right)(x)= & -\int k_{0}(\tau, x-y)\left[-m+\Phi\left(\frac{x+y}{2}\right)\right] g(y) d y .
\end{aligned}
$$

We shall show that there is a constant $C>0$ such that

$$
\left\|I_{1}(\tau) g+H_{A} g\right\|_{0} \leqq C\|g\|_{1},
$$

and

$$
\left\|I_{2}(\tau) g+[-m+\Phi] g\right\|_{0} \leqq C\|g\|_{1},
$$

uniformly in $0<\tau<1$ for every $g \in \mathscr{S}\left(\mathbb{R}^{d}\right)$, and that for each fixed $g \in \mathscr{S}\left(\mathbb{R}^{d}\right)$,

$$
\left\|I_{1}(\tau) g+H_{A} g\right\|_{0} \rightarrow 0,
$$

and

$$
\left\|I_{2}(\tau) g+[-m+\Phi] g\right\|_{0} \rightarrow 0
$$

as $\tau \downarrow 0$.

Proof of (4.11) and (4.12) . For the term involving the pseudo-differential operator $\left(-\Delta_{y}+m^{2}\right)^{1 / 2}$ in (4.9) or (4.10) we have as oscillatory integrals

$$
\begin{aligned}
\left(-\Delta_{y}+m^{2}\right)^{1 / 2}\left(\exp \left[i A\left(\frac{x+y}{2}\right) \cdot(x-y)-\Phi\left(\frac{x+y}{2}\right) \tau\right] g(y)\right) \\
\equiv(2 \pi)^{-d} \iint e^{i\left(y-y^{\prime}\right) \cdot p}\left(p^{2}+m^{2}\right)^{1 / 2} \\
\quad \cdot \exp \left[i A\left(\frac{x+y^{\prime}}{2}\right) \cdot\left(x-y^{\prime}\right)-\Phi\left(\frac{x+y^{\prime}}{2}\right) \tau\right] g\left(y^{\prime}\right) d y^{\prime} d p \\
=(2 \pi)^{-d} \iint e^{i\left(y-y^{\prime}\right) \cdot p} \exp \left[i A\left(\frac{y+y^{\prime}}{2}\right) \cdot(x-y)-\Phi\left(\frac{x+y^{\prime}}{2}\right) \tau\right] \\
\quad \times\left[\left(p-A\left(\frac{x+y^{\prime}}{2}\right)\right)^{2}+m^{2}\right]^{1 / 2} g\left(y^{\prime}\right) d y^{\prime} d p .
\end{aligned}
$$

To see (4.13) let $\chi$ be a function in $\mathscr{S}\left(\mathbb{R}^{d}\right)$ with $\chi(0)=1$. For every multi-index $\alpha$ there exists a constant $C_{\alpha}>0$ independent of $0<\varepsilon<1$ such that

$$
\left|\partial_{x}^{\alpha} \chi(\varepsilon x)\right| \leqq C_{\alpha} \varepsilon^{\sigma}\left(1+x^{2}\right)^{-(|\alpha|-\sigma) / 2}, \quad 0 \leqq \sigma \leqq|\alpha| .
$$

As $\varepsilon \downarrow 0, \chi(\varepsilon x)$ converges to 1 uniformly on compact sets in $\mathbb{R}^{d}$ and $\partial_{x}^{\alpha} \chi(\varepsilon x)$ with $|\alpha| \neq 0$ converges to zero uniformly in $\mathbb{R}^{d}$. Then the integral in the second member of (4.13) is by definition

$$
\lim _{\epsilon \rightarrow 0} \iint e^{i\left(y-y^{\prime}\right) \cdot p} \chi(\varepsilon p)\left(p^{2}+m^{2}\right)^{1 / 2} \exp \left[i A\left(\frac{x+y^{\prime}}{2}\right) \cdot\left(x-y^{\prime}\right)-\Phi\left(\frac{x+y^{\prime}}{2}\right) \tau\right] g\left(y^{\prime}\right) d y^{\prime} d p .
$$


By the change of variables $p^{\prime}=p+A\left(\frac{1}{2}\left(x+y^{\prime}\right)\right)$ and by integration by parts this equals (with $p$ again instead of $p^{\prime}$ )

$$
\begin{aligned}
\lim _{\varepsilon \rightarrow 0} \iint e^{i\left(y-y^{\prime}\right) \cdot p} \chi\left(\varepsilon\left(p-A\left(\frac{x+y^{\prime}}{2}\right)\right)\right) \exp \left[i A\left(\frac{x+y^{\prime}}{2}\right) \cdot(x-y)-\Phi\left(\frac{x+y^{\prime}}{2}\right) \tau\right] \\
\times\left[\left(p-A\left(\frac{x+y^{\prime}}{2}\right)\right)^{2}+m^{2}\right]^{1 / 2} g\left(y^{\prime}\right) d y^{\prime} d p \\
=\lim _{\varepsilon \rightarrow 0} \iint e^{i\left(y-y^{\prime}\right) \cdot p\left(1+p^{2}\right)^{-l}\left(1-\Delta_{y^{\prime}}\right)^{l}} \\
\quad \times\left\{\chi\left(\varepsilon\left(p-A\left(\frac{x+y^{\prime}}{2}\right)\right)\right) \exp \left[i A\left(\frac{x+y^{\prime}}{2}\right) \cdot(x-y)-\Phi\left(\frac{x+y^{\prime}}{2}\right) \tau\right]\right. \\
\left.\quad \times\left[\left(p-A\left(\frac{x+y^{\prime}}{2}\right)\right)^{2}+m^{2}\right]^{1 / 2} g\left(y^{\prime}\right)\right\} d y^{\prime} d p,
\end{aligned}
$$

where $l$ is an integer $>d / 2$, and note

$$
e^{-i y^{\prime} \cdot p}=\left(1+p^{2}\right)^{-l}\left(1-\Delta_{y^{\prime}}\right)^{l} e^{-i y^{\prime} \cdot p} \text {. }
$$

Since the derivatives of $A(x)$ are bounded, $\chi\left(\varepsilon\left(p-A\left(\frac{1}{2}\left(x+y^{\prime}\right)\right)\right)\right)$ converges to 1 uniformly on compact sets in both $p$ and $y^{\prime}$, and $\partial_{v^{\prime}}^{\alpha} \chi\left(\varepsilon\left(p-A\left(\frac{1}{2}\left(x+y^{\prime}\right)\right)\right)\right)$ with $|\alpha| \neq 0$ converges to zero uniformly in both $p$ and $y^{\prime}$. It follows that the last integral above is equal to

$$
\begin{aligned}
& \iint e^{i\left(y-y^{\prime}\right) \cdot p}\left(1+p^{2}\right)^{-l}\left(1-\Delta_{y^{\prime}}\right)^{l}\left\{\exp \left[i A\left(\frac{x+y^{\prime}}{2}\right) \cdot(x-y)-\Phi\left(\frac{x+y^{\prime}}{2}\right) \tau\right]\right. \\
& \left.\quad \times\left[\left(p-A\left(\frac{x+y^{\prime}}{2}\right)\right)^{2}+m^{2}\right]^{1 / 2} g\left(y^{\prime}\right)\right\} d y^{\prime} d p \\
& =\lim _{\varepsilon \rightarrow 0} \iint e^{i\left(y-y^{\prime}\right) \cdot p} \chi(\varepsilon p)\left(1+p^{2}\right)^{-l}\left(1-\Delta_{y^{\prime}}\right)^{l}\{\cdots\} d y^{\prime} d p \\
& =\lim _{\varepsilon \rightarrow 0} \iint e^{i\left(y-y^{\prime}\right) \cdot p} \chi(\varepsilon p)\left\{\exp \left[i A\left(\frac{x+y^{\prime}}{2}\right) \cdot(x-y)-\Phi\left(\frac{x+y^{\prime}}{2}\right) \tau\right] g\left(y^{\prime}\right)\right\} d y^{\prime} d p .
\end{aligned}
$$

Here the last equality above is due to integration by parts, and the last member is nothing but the integral in the last member of (4.13).

On the other hand, (1.3) is invariant under any translation $y \mapsto y+z$ of the integration variable, so that

$$
\left(H_{A} g\right)(x)=(2 \pi)^{-d} \iint e^{i\left(x-y^{\prime}-z\right) \cdot p}\left(\left(p-A\left(\frac{x+y^{\prime}+z}{2}\right)\right)^{2}+m^{2}\right)^{1 / 2} g\left(y^{\prime}+z\right) d y^{\prime} d p .
$$

It follows that

$$
\begin{aligned}
& \left(I_{1}(\tau) g\right)(x)+\left(H_{A} g\right)(x) \\
& \quad=(2 \pi)^{-d} \int k_{0}(\tau, x-y) d y \iint e^{i\left(y-y^{\prime}\right) \cdot p}
\end{aligned}
$$




$$
\begin{aligned}
& \times\left\{\exp \left[i A\left(\frac{x+y^{\prime}}{2}\right) \cdot(x-y)-\Phi\left(\frac{x+y^{\prime}}{2}\right) \tau\right]\right. \\
& \cdot\left[\left(p-A\left(\frac{x+y^{\prime}}{2}\right)\right)^{2}+m^{2}\right]^{1 / 2} g\left(y^{\prime}\right) \\
& \left.-\left[\left(p-A\left(\frac{x+y^{\prime}+(x-y)}{2}\right)\right)^{2}+m^{2}\right]^{1 / 2} g\left(y^{\prime}+(x-y)\right)\right\} d y^{\prime} d p .
\end{aligned}
$$

We use the Schwarz inequality with property $(4.3)$ of $k_{0}(\tau, x)$ to estimate the $L^{2}$ norm of (4.14) and make the change of variables $x-y=z$ to get

$$
\begin{aligned}
\left\|I_{1}(\tau) g+H_{A} g\right\|_{0}^{2} \leqq & (2 \pi)^{-2 d} \int d z k_{0}(\tau, z) \int \mid \iint e^{i\left(y-y^{\prime}\right) \cdot p} \\
& \times\left\{\exp \left[i A\left(\frac{y+y^{\prime}+z}{2}\right) \cdot z-\Phi\left(\frac{y+y^{\prime}+z}{2}\right) \tau\right]\right. \\
& \cdot\left[\left(p-A\left(\frac{y+y^{\prime}+z}{2}\right)\right)^{2}+m^{2}\right]^{1 / 2} g\left(y^{\prime}\right) \\
& \left.-\left[\left(p-A\left(\frac{y+y^{\prime}+2 z}{2}\right)\right)^{2}+m^{2}\right]^{1 / 2} g\left(y^{\prime}+z\right)\right\}\left.d y^{\prime} d p\right|^{2} d y \\
= & \int d z k_{0}(\tau, z) \int \mid\left(Q_{1}\left(Y, D_{y}, Y^{\prime} ; z, \tau\right) g\right)(y) \\
& +\left.\left(Q_{2}\left(Y, D_{y}, Y^{\prime} ; z\right)\left(g-g^{z}\right)\right)(y)\right|^{2} d y,
\end{aligned}
$$

where $g^{z}(y)=g(y+z) . \quad Q_{1}\left(Y, D_{y}, Y^{\prime} ; z, \tau\right)$ and $Q_{2}\left(Y, D_{y}, Y^{\prime} ; z\right)$ are the pseudodifferential operators with multiple symbols (see [19])

$$
\begin{aligned}
q_{1}(z, \tau) \equiv q_{1}\left(y, p, y^{\prime} ; z, \tau\right)= & \exp \left[i A\left(\frac{y+y^{\prime}+z}{2}\right) \cdot z-\Phi\left(\frac{y+y^{\prime}+z}{2}\right) \tau\right] \\
& \times\left[\left(p-A\left(\frac{y+y^{\prime}+z}{2}\right)\right)^{2}+m^{2}\right]^{1 / 2} \\
& -\left[\left(p-A\left(\frac{y+y^{\prime}+2 z}{2}\right)\right)^{2}+m^{2}\right]^{1 / 2}
\end{aligned}
$$

and

$$
q_{2}(z) \equiv q_{2}\left(y, p, y^{\prime} ; z\right)=\left[\left(p-A\left(\frac{y+y^{\prime}+2 z}{2}\right)\right)^{2}+m^{2}\right]^{1 / 2}=h_{A}\left(p, \frac{y+y^{\prime}+2 z}{2}\right),
$$

where $z$ and $\tau$ are parameters. It is easy to see that for all multi-indices $\alpha, \beta$ and $\beta^{\prime}$ there are constants $C_{1, \alpha \beta \beta^{\prime}}$ and $C_{2, \alpha \beta \beta^{\prime}}$ such that

$$
\left|\partial_{p}^{\alpha} \partial_{y}^{\beta} \partial_{y^{\prime}}^{\beta^{\prime}} q_{1}\left(y, p, y^{\prime} ; z, \tau\right)\right| \leqq C_{1, \alpha \beta \beta^{\prime}}(|z|+\tau)(1+|z|+\tau)^{|\beta|+\left|\beta^{\prime}\right|}\left(1+p^{2}\right)^{(1-|\alpha|) / 2},
$$

and

$\left|\partial_{p}^{\alpha} \partial_{y}^{\beta} \partial_{y^{\prime}}^{\beta^{\prime}} q_{2}\left(y, p, y^{\prime} ; z\right)\right| \leqq C_{2, \alpha \beta \beta^{\prime}}\left(1+p^{2}\right)^{(1-|\alpha|) / 2}, \quad p \in \mathbb{R}^{d},\left(y, y^{\prime}\right) \in \mathbb{R}^{d} \times \mathbb{R}^{d}, z \in \mathbb{R}^{d}, \tau>0$. 
By the Calderón-Vaillancourt theorem $([2,18])$ there exist a constant $C>0$ and integers $k, l \geqq 0$ such that

$$
\left\|Q_{1}\left(Y, D_{y}, Y^{\prime} ; z, \tau\right) g\right\|_{0} \leqq C\left|q_{1}(z, \tau)\right|_{k, l}^{(1)}\|g\|_{1},
$$

and

$$
\left\|Q_{2}\left(Y, D_{y}, Y^{\prime} ; z\right) g\right\|_{0} \leqq C\left|q_{2}(z)\right|_{k, l}^{(1)}\|g\|_{1}
$$

for $g \in \mathscr{S}\left(\mathbb{R}^{d}\right)$. Here

$$
\left|q_{1}(z, \tau)\right|_{k, l}^{(1)}=C_{1}(|z|+\tau)(1+|z|+\tau)^{l} \quad \text { with } \quad C_{1}=\max _{|\alpha| \leqq k,|\beta|+\left|\beta^{\prime}\right| \leqq l} \inf C_{1, \alpha \beta \beta^{\prime}} \text { of }(4.15),
$$

and

$$
\left|q_{2}(z)\right|_{k, l}^{(1)}=\max _{|\alpha| \leqq k,|\beta|+\left|\beta^{\prime}\right| \leqq l} \inf C_{2, \alpha \beta \beta^{\prime}} \text { of }(4.16)
$$

is bounded by a constant $C_{2}$ independent of $z$ (Inspection with [19, Chap. 7, Theorem 2.4] or [18, Theorem 2.2]) gives that it suffices if $k \geqq 4[d / 2+1]$ and $l \geqq 2[d / 2+1]+2[(d+1) / 2+1$.

Putting all this together we have with some constants $C_{3}$ and $C_{4}$

$$
\begin{aligned}
\left\|I_{1}(\tau) g+H_{A} g\right\|_{0}^{2} \leqq & C_{3} \int d z k_{0}(\tau, z)\left[(|z|+\tau)(1+|z|+\tau)^{l}\|g\|_{1}+\left\|g-g^{z}\right\|_{1}\right]^{2} \\
\leqq & C_{4} \int k_{0}(\tau, z)\left(|z|^{2}+\tau^{2}\right)\left(1+|z|^{2 l}+\tau^{2 l}\right) d z\|g\|_{1}^{2} \\
& +2 C_{3} \int k_{0}(\tau, z)\left\|g-g^{z}\right\|_{1}^{2} d z
\end{aligned}
$$

Thus we shall get (4.11) and (4.12) , if the following lemma is shown. We shall continue to put $c=e=1$.

Lemma 4.3. (i) For $n$ a positive integer

$$
\int_{\mathbb{R}^{d}} k_{0}(\tau, z)|z|^{2 n} d z \rightarrow 0, \quad \text { as } \quad \tau \downarrow 0 .
$$

(ii)

$$
\int_{\mathbb{R}^{d}} k_{0}(\tau, z)\left\|g-g^{z}\right\|_{1}^{2} d z \rightarrow 0, \quad \text { as } \quad \tau \downarrow 0,
$$

for $g \in H^{1}\left(\mathbb{R}^{d}\right)$, where $g^{z}(y)=g(y+z)$.

Proof. (i) We have with (4.2),

$$
\begin{aligned}
\int_{\mathbb{R}^{d}} k_{0}(\tau, z)|z|^{2 n} d z= & 2^{-(d-1) / 2} \pi^{-(d+1) / 2} m^{(d+1) / 2} e^{m \tau} \tau \int_{\mathbb{R}^{d}}|x|^{m+d-1} \\
& \cdot\left(x^{2}+\tau^{2}\right)^{-(d+1) / 4} K_{(d+1) / 2}\left(m\left(x^{2}+\tau^{2}\right)^{1 / 2}\right) d x \\
= & c_{d, n} m^{1 / 2-n} \tau^{1 / 2+n} e^{m \tau} K_{n-1 / 2}(m \tau) \\
\leqq & C(d, n, m) \tau, \text { as } \tau \downarrow 0,
\end{aligned}
$$

with constants $c_{d, n}$ depending on $d$ and $n$ and $C(d, n, m)$ depending on $d, n$ and $m$, but independent of $\tau$ (use formulas for Bessel functions [5, Chap. VII, 7.14.2, (50), p. 95, and 7.2.6, (40), p. 10]). 
(ii) First we claim that for every $\varepsilon>0$ there exists a constant $R_{0}>0$ such that for $R \geqq R_{0}$.

$$
\int_{|z|>R} k_{0}(\tau, \tau z) \tau^{d} d z<\varepsilon \quad \text { uniformly in } 0<\tau<1 .
$$

To see this let $\chi$ be a nonnegative $C^{\infty}$ function such that $\chi(x)=1$ if $|x| \leqq 1 / 2$, and $\chi(x)=0$ if $|x| \geqq 1$. Put $\chi_{R}(x)=\chi(x / R)$. Then

$$
\begin{aligned}
\int_{|z|>R} k_{0}(\tau, \tau z) \tau^{d} d z & \leqq \int\left(1-\chi_{R}(z)\right) k_{0}(\tau, \tau z) \tau^{d} d z \\
& =1-(2 \pi)^{-d / 2} \int \hat{\chi}_{R}(p) \exp \left\{-\tau\left[\left(p^{2} / \tau^{2}+m^{2}\right)^{1 / 2}-m\right]\right\} d p
\end{aligned}
$$

where $\hat{\chi}_{R}$ is the Fourier transform of $\chi_{R}$ and equal to $R^{d} \hat{\chi}(R p)$. It follows that (4.18) is smaller than or equal to

$$
\begin{aligned}
1- & (2 \pi)^{-d / 2} \int R^{d} \hat{\chi}(R p) \exp \left\{-\left[\left(p^{2}+m^{2} \tau^{2}\right)^{1 / 2}-m \tau\right]\right\} d p \\
& \left.=1-(2 \pi)^{-d / 2} \int \hat{\chi}(p) \exp \left\{-\left[(p / R)^{2}+m^{2} \tau^{2}\right)^{1 / 2}-m \tau\right]\right\} d p,
\end{aligned}
$$

which tends to zero as $R \rightarrow \infty$ uniformly in $0<\tau<1$, by the Lebesgue dominated convergence theorem. Our claim has thus been shown.

We are now in a position to prove the second statement of Lemma 4.3. Let $\varepsilon>0$. Choose $R_{0}>0$ such that for $R \geqq R_{0}$ (4.17) holds uniformly in $0<\tau<1$. Putting $z=\tau z^{\prime}$ (writing $z$ again instead of $z^{\prime}$ ) we have

$$
\int k_{0}(\tau, z)\left\|g-g^{z}\right\|_{1}^{2} d z=\int \tau^{d} k_{0}(\tau, \tau z)\left\|g-g^{\tau z}\right\|_{1}^{2} d z=\int_{|z| \leqq R}+\int_{|z|>R} .
$$

If $R \geqq R_{0}$, the second term above is smaller than or equal to $4 \varepsilon\|g\|_{1}^{2}$. The first term tends to zero as $\tau \downarrow 0$, because $\left\|g-g^{\tau z}\right\|_{1}^{2} \rightarrow 0$ as $\tau \downarrow 0$ by the Lebesgue theorem, and because $\tau^{d} k_{0}(\tau, \tau z) \rightarrow C(d)\left(z^{2}+1\right)^{-(d+1) / 2}$ as $\tau \downarrow 0$ with a constant $C(d)$ depending on $d$, since $K_{(d+1) / 2}(r)=C(d) 2^{(d-1) / 2} \pi^{(d+1) / 2} r^{-(d+1) / 2}+O\left(r^{1-(d+1) / 2}\right)$ as $r \rightarrow 0$ as seen from (4.2) in Lemma 4.1 (use [5, Chap. VII, 7.2.6, (40), p. 10, and 7.2.5, (37), p.9]). This proves Lemma 4.3.

Proof of $(4.11)_{\mathrm{II}}$ and (4.12) $)_{\mathrm{II}}$. This case is simpler. We have by the Schwarz inequality and the property (4.3) of $k_{0}(\tau, x)$,

$$
\begin{aligned}
\left\|I_{2}(\tau) g+[-m+\Phi] g\right\|_{0}^{2} \leqq & \int \mathrm{d} x \int k_{0}(\tau, x-y) \mid\left(m-\Phi\left(\frac{x+y}{2}\right)\right) \\
& \times \exp \left[i A\left(\frac{x+y}{2}\right) \cdot(x-y)-\Phi\left(\frac{x+y}{2}\right) \tau\right] g(y) \\
& -\left.(m-\Phi(x)) g(x)\right|^{2} d y \\
\leqq & \int d z k_{0}(\tau, z) \int \mid\{(m-\Phi(y+z / 2)) \exp [i A(y+z / 2) z \\
& -\Phi(y+z / 2) \tau]-(m-\Phi(y+z))\} g(y) \\
& +\left.(m-\Phi(y+z))\left(g(y)-g^{z}(y)\right)\right|^{2} d y .
\end{aligned}
$$


Then there exists a constant $C>0$ such that

$$
\left\|I_{2}(\tau) g+[-m+\Phi] g\right\|_{0}^{2} \leqq C \int k_{0}(\tau, z)(|z|+\tau)^{2} d z\|g\|_{1}^{2}+C \int k_{0}(\tau, z)\left\|g-g^{z}\right\|_{1}^{2} d z .
$$

The remaining proof proceeds in the same way as the last part of the proof of $(4.11)_{I}$ and (4.12). This ends the proof of Lemma 4.12.

4.3. The Path Integral Representation (2.1). We shall now investigate what is the limit of the right-hand side of (4.6) to establish the path integral formula (2.1) with (2.2).

We do first for $g \in L^{2}\left(\mathbb{R}^{d}\right)$ which is bounded and continuous. Then we obtain from (4.6) with (4.7), using the path space measure $\lambda_{0, x}$ introduced in Sect.4.1,

$$
\left(T(t / n)^{n} g\right)(x)=\int e^{-S_{n}(X)} g(X(t)) d \lambda_{0, x}(X) .
$$

Here $S_{n}(X)=S_{n 1}(X)+S_{n 2}(X)$,

$$
\begin{aligned}
& S_{n 1}(X)=i \sum_{j=1}^{n}(e / c) A\left(\frac{1}{2}\left(X\left(t_{j-1}\right)+X\left(t_{j}\right)\right)\right) \cdot\left(X\left(t_{j}\right)-X\left(t_{j-1}\right)\right), \\
& S_{n 2}(X)=\sum_{j=1}^{n} e \Phi\left(\frac{1}{2}\left(X\left(t_{j-1}\right)+X\left(t_{j}\right)\right)\right)\left(t_{j}-t_{j-1}\right)
\end{aligned}
$$

with $t_{j}=j t / n$.

As for $S_{n 2}(X)$ in (4.20), it is evident that for each $X$ in $D_{0, x}\left([0, \infty) \rightarrow \mathbb{R}^{d}\right), S_{n 2}(X)$ converges to $\int_{0}^{t} e \Phi(X(s)) d s$ as $n \rightarrow \infty$. To see the convergence of $S_{n 1}(X)$, introduce the functional

$$
F_{j}(X(s))=i(e / c) A\left(\frac{1}{2}\left(X(s)+X\left(t_{j-1}\right)\right)\right) \cdot\left(X(s)-X\left(t_{j-1}\right)\right)
$$

of the path $X(s)$ in $D_{0, x}\left([0, \infty) \rightarrow \mathbb{R}^{d}\right)$. Note the Lévy-Itô theorem (2.3) to apply Itô's formula [16, Chap. II, 5, Theorem 5.1] to each $F_{j}(X(s))=F_{j}(X(s))-F_{j}\left(X\left(t_{j-1}\right)\right)$. Then we have with $\left(\mathbb{R}^{d}\right)^{\times} \equiv \mathbb{R}^{d} \backslash\{0\}$,

$$
\begin{aligned}
& S_{n 1}(X)=\int_{0}^{t+} \int_{\left(\mathbb{R}^{d}\right)^{d} \times} \sum_{j=1}^{n} I_{\left(t_{j-1}, t_{j}\right]}(s)\left[F\left(X(s-)+y I_{\{|y| \geq 1\}}(y)\right)-F(X(s-))\right] N_{X}(d s d y) \\
& +\int_{0}^{t+} \int_{\left(\mathbb{R}^{d}\right) \times} \sum_{j=1}^{n} I_{\left(t_{j-1}, t_{j}\right]}(s)\left[F\left(X(s-)+y I_{\{|y|<1\}}(y)\right)-F(X(s-))\right] \tilde{N}_{X}(d s d y) \\
& +\int_{0}^{t} \int_{\left(\mathbb{R}^{d}\right)^{\infty} \times} \sum_{j=1}^{n} I_{\left(t_{j-1}, t_{j}\right]}(s)\left[F\left(X(s)+y I_{\{|y|<1\}}(y)\right)-F(X(s))\right. \\
& \left.-I_{\{|y|<1\}}(y)(y \cdot \partial) F(X(s))\right] \hat{N}(d s d y) \\
& =i \int_{0}^{t+} \int_{\left(\mathbb{R}^{d}\right)^{d}} L_{n 1}(s, y, X) N_{X}(d s d y)+i \int_{0}^{t+} \int_{\left(\mathbb{R}^{d}\right)^{d}} L_{n 2}(s, y, X) \tilde{N}_{X}(d s d y) \\
& +i \int_{0_{\left(\mathbb{R}^{d}\right)^{d}}^{t}}^{t} L_{n 3}(s, y, X) \hat{N}(d s d y) \text {. }
\end{aligned}
$$

Here

$$
\begin{aligned}
L_{n 1}(s, y, X)= & \sum_{j=1}^{n} I_{\left(t_{j-1}, t_{j}\right]}(s)(e / c)\left[A\left(\frac{1}{2}\left(X(s-)+X\left(t_{j-1}\right)\right)+y I_{\{|y| \geqq 1\}}(y)\right)\right. \\
& \cdot\left(X(s-)-X\left(t_{j-1}\right)+y I_{\{|y| \geq 1\}}(y)\right) \\
& \left.-A\left(\frac{1}{2}\left(X(s-)+X\left(t_{j-1}\right)\right)\right) \cdot\left(X(s-)-X\left(t_{j-1}\right)\right)\right],
\end{aligned}
$$




$$
\begin{aligned}
L_{n 2}(s, y, X)= & \sum_{j=1}^{n} I_{\left\langle t_{j-1}, t_{j}\right]}(s)(e / c)\left[A \left(\frac{1}{2}\left(X(s-)+X\left(t_{j-1}\right)+y I_{\{|y|<1\}}(y)\right)\right.\right. \\
& \cdot\left(X(s-)-X\left(t_{j-1}\right)+y I_{\{|y|<1\}}(y)\right) \\
& \left.-A\left(\frac{1}{2}\left(X(s-)+X\left(t_{j-1}\right)\right)\right) \cdot\left(X(s-)-X\left(t_{j-1}\right)\right)\right] \\
L_{n 3}(s, y, X)= & \sum_{j=1}^{n} I_{\left(t_{j-1}, t_{j}\right]}(s)(e / c)\left\{A ( \frac { 1 } { 2 } ( X ( s ) + X ( t _ { j - 1 } ) + y I _ { \{ | y | < 1 \} } ( y ) ) ) \cdot \left(X(s)-X\left(t_{j-1}\right)\right.\right. \\
& \left.+y I_{\{|y|<1\}}(y)\right)-A\left(\frac{1}{2}\left(X(s)+X\left(t_{j-1}\right)\right)\right) \cdot\left(X(s)-X\left(t_{j-1}\right)\right) \\
& -I_{\{|y|<1\}}(y)\left[(y) \cdot \partial\left(\frac{1}{2}\left(X(s)+X\left(t_{j-1}\right)\right)\right) \cdot\left(X(s)-X\left(t_{j-1}\right)\right)\right. \\
& \left.\left.+y \cdot A\left(\frac{1}{2}\left(X(s)+X\left(t_{j-1}\right)\right)\right)\right]\right\} .
\end{aligned}
$$

Now we take $n=2^{k}$, so that $t_{j}=2^{-k} j t, j=0,1, \ldots, 2^{k}$. Then we see for each fixed $X$ in $D_{0, x}\left([0, \infty) \rightarrow \mathbb{R}^{d}\right)$ that

$$
\begin{aligned}
& L_{n 1}(s, y, X) \rightarrow(e / c) A(X(s-)+y / 2) \cdot y I_{\{|y| \geq 1\}}(y), \\
& L_{n 2}(s, y, X) \rightarrow(e / c) A(X(s-)+y / 2) \cdot y I_{\{|y|<1\}}(y), \\
& L_{n 3}(s, y, X) \rightarrow(e / c)(A(X(s)+y / 2)-A(X(s))] \cdot y I_{\{|y|<1\}}(y),
\end{aligned}
$$

as $k \rightarrow \infty$. It follows that when $k \rightarrow \infty$, the three integrals of $L_{n 1}, L_{n 2}$ and $L_{n 3}$ in $S_{n 1}(X)$ approach the first three integrals in the second member of (2.2). Therefore an application of the Lebesgue dominated convergence theorem shows the right-hand side of (4.19) approaches the right-hand side of (2.1). This was when $g$ is in $L^{2}\left(\mathbb{R}^{d}\right)$, bounded and continuous.

When $g \in L^{2}\left(\mathbb{R}^{d}\right)$ in general, there exists a sequence $\left\{g_{n}\right\}$ of bounded continuous functions in $L^{2}\left(\mathbb{R}^{d}\right)$ such that $\left|g_{n}(x)\right| \leqq|g(x)|$, a.e., $n=1,2, \ldots$, and $g_{n} \rightarrow g$ in $L^{2}\left(\mathbb{R}^{d}\right)$ as $n \rightarrow \infty$. By taking a subsequence if necessary, $g_{n} \rightarrow g$ a.e. Then it holds that $\int g_{n}(X(t)) d \lambda_{0, x}(X)=\int k_{0}(t, x-y) g_{n}(y) d y$, and so, by the Lebesgue dominated convergence theorem, for $g$ in place of $g_{n}$. It follows that (2.1) holds for $g$. This completes the proof of Theorem 2.1(ii).

4.4. Proof of Theorem 2.1(iii). From the path integral formula (2.1) with $\Phi \equiv 0$ we have

$$
\begin{aligned}
\left(\exp \left[-t\left(H_{A}-m c^{2}\right)\right] g, g\right) & \leqq \iint|g(X(t))||g(x)| d \lambda_{0, x}(X) d x \\
& =\left(\exp \left[-t\left(H_{0}-m c^{2}\right)\right]|g|,|g|\right) \leqq\|g\|^{2}
\end{aligned}
$$

for $g \in L^{2}\left(\mathbb{R}^{d}\right)$. It follows that the selfadjoint operator $\exp \left[-t\left(H_{A}-m c^{2}\right)\right]$ is a contraction, so that $H_{A}-m c^{2}$ is nonnegative. This ends the proof of Theorem 2.1.

\section{Nonregular and Unbounded Vector and Scalar Potentials}

In the previous sections we have been assuming regularity and boundedness of both vector and scalar potentials $A$ and $\Phi$. However, in application nonregular and unbounded $A$ and $\Phi$ appear. For instance, the Coulomb potential $\Phi(x)$ has the $|x|^{-1}$ singularity (cf. $[11,3,4]$ ), and the vector potential $A(x)$ of the constant magnetic field is linear in $x$. 
If $\Phi(x)$ is measurable in $\mathbb{R}^{d}$, and if $e \Phi=e \Phi_{+}-e \Phi_{-}$with $e \Phi_{+} \geqq 0, e \Phi_{-} \geqq 0$, where $\Phi_{+} \in L_{\text {loc }}^{1}\left(\mathbb{R}^{d} \backslash G\right)$ with $G$ a closed subset of measure zero, and for some constants $0<\delta<1$ and $C \geqq 0$,

$$
\int e \Phi_{-}(x)|f(x)|^{2} \mathrm{~d} x \leqq \delta\left\|\left(-c^{2} \Delta+m^{2} c^{4}\right)^{1 / 4} f\right\|^{2}+C\|f\|^{2},
$$

for all $f \in H^{1 / 2}\left(\mathbb{R}^{d}\right)$, then the path integral formula (2.1) with (2.2) for the form sum $H=H_{A}+e \Phi$ will be shown in the same way as [27, Chap. II, Theorem 6.2]. Recall Gårding's inequality (3.2).

As far as $A$ is concerned, we have only to assume $A(x)$ has bounded, continuous derivatives up to sufficiently high order. A further reduction of regularity of $A$ will be done with the theory of pseudo-differential operators with nonregular symbols (Nagase [24,23]). It will be also possible to include the unbounded case where $A(x)$ is an $\mathbb{R}^{d}$-valued function of polynomial growth.

It will suffice to assume, as $S(t, 0 ; X)$ in (2.2) suggests, that $A$ is Hölder-continuous of order $\alpha, 0<\alpha \leqq 1$, i.e. for every $R>0$ there exists a constant $C>0$ such that

$$
|A(x+z)-A(x)| \leqq C|z|^{\alpha}, \quad|z|<1, \quad|x| \leqq R .
$$

In fact, we see with (2.6) and (2.8) that we may regard $|x|^{2} n(d x)$ as a measure on $\mathbb{R}^{d}$. Let $\varepsilon>0$, and $\beta=(d-1) / 2+\varepsilon$. Apply the pseudo-differential operator $\left(-\Delta_{p}\right)^{\beta}$ to both sides of (2.7) with $k=1$. Then we have for some constant $C>0$,

$$
\left.\left|\int_{\mathbb{R}^{d}} e^{i p \cdot y}\right| y\right|^{2+2 \beta} n(d y)|=|\left(-\Delta_{p}\right)^{1+\beta}\left(c^{2} p^{2}+m^{2} c^{4}\right)^{1 / 2} \mid \leqq C\left(1+p^{2}\right)^{-\beta-1 / 2},
$$

which is in $L^{1}\left(\mathbb{R}^{d}\right)$ with respect to $p$. Therefore there exists a function $\rho(y)$ in $L^{\infty}\left(\mathbb{R}^{d}\right)$ such that $|y|^{2+2 \beta} n(d y)=\rho(y) d y$. Since $\hat{N}(d s d y)=d s n(d y)$, the condition (5.2) is then obtained from this and (2.2).

\section{Heuristic Remark}

In this section we shall heuristically see our method is nothing but a rigorous application of the phase space path integral or Hamiltonian path integral with the "midpoint" prescription $([21,22,7])$.

Let $f, g \in L^{2}\left(\mathbb{R}^{d}\right)$. Then $\left(g, e^{-i t H} f\right)$ is the transition amplitude that a Weyl quantized charged particle (with the Hamiltonian $h(p, x)$ in (1.2)) at state $f$ at time 0 will be at state $g$ at time $t$. The phase space path integral or Hamiltonian path integral method assumes that it is given by a formal "integral"

$$
\left(g, e^{-i t H} f\right)=\int \overline{g(X[t])} e^{i S(t, 0 ; P, X)} f(X[0]) D(P) D(X),
$$

where $S(t, 0 ; P, X)$ is the action which is defined with (1.2) by

$$
S(t, 0 ; P, X)=\int_{0}^{t}[P[s] \cdot \dot{X}[s]-h(P[s], X[s])] d s,
$$

where $X[s]$ and $P[s]$ are the position and momentum. $D(P) D(X)$ is a formal "measure" $\prod_{0 \leqq s \leqq t}(2 \pi)^{-d} d P[s] d X[s]$ on the space of the phase space paths $(P[s], X[s])$. In this formal phase space path "integral" we first make an analytic 
continuation which replaces $t$ by $-i t$ to go from $e^{-i t H}$ to $e^{-t H}$, and put $P(s)=P[-i s]$, $X(s)=X[-i s]$. Using the substitutions $d s \rightarrow-i d s, \dot{X}[s]=(d / d s) X[s] \rightarrow i \dot{X}(s)$, we are led to the formal expression

$$
\begin{aligned}
\left(g, e^{-t H} f\right)= & \int \overline{g(X(t))} \exp \left\{\int_{0}^{t}[i P(s) \cdot \dot{X}(s)-h(P(s), X(s))] d s\right\} f(X(0)) \\
& \times \prod_{0 \leqq s \leqq t}(2 \pi)^{-d} d P(s) d X(s) .
\end{aligned}
$$

Next we make the change of variables: $X^{\prime}(s)=X(s), P^{\prime}(s)=P(s)-(e / c) A(X(s))$. Then we obtain from (6.3) (writing $(P(s), X(s))$ again instead of $\left.\left(P^{\prime}(s), X^{\prime}(s)\right)\right)$

$$
\begin{aligned}
\left(e^{-t H} g, f\right)=\left(g, e^{-t H} f\right)= & \int \overline{g(X(t))} \exp \left\{\int_{0}^{t}[i(P(s)+(e / c) A(X(s))) \dot{X}(s)\right. \\
& \left.\left.-\left(c^{2} P(s)^{2}+m^{2} c^{4}\right)^{1 / 2}-e \Phi(X(s))\right] d s\right\} f(X(0)) \\
& \cdot \prod_{0 \leqq s \leqq t}(2 \pi)^{-d} d P(s) d X(s) .
\end{aligned}
$$

We understand that the last member of (6.4) is defined with a time division procedure and the position-dependent terms are evaluated at the midpoints (see [21]). Then

$$
\begin{aligned}
\left(e^{-t H} g, f\right)= & \lim _{n \rightarrow \infty} \int_{\int_{\mathbb{R}^{2 d}} \cdots \int_{\mathbb{R}^{2 d}}^{n} \int} \int_{\mathbb{R}^{d}} \overline{g\left(x^{(n)}\right)} \exp \left\{\sum _ { j = 1 } ^ { n } \left[i\left(p^{(j-1)}+(e / c) A\left(\frac{x^{(j-1)}+x^{(j)}}{2}\right)\right)\right.\right. \\
& \cdot\left(x^{(j)}-x^{(j-1)}\right) /\left(t_{j}-t_{j-1}\right)-\left(c^{2}\left(p^{(j-1)}\right)^{2}+m^{2} c^{4}\right)^{1 / 2} \\
& \left.\left.-e \Phi\left(\frac{x^{(j-1)}+x^{(j)}}{2}\right)\right]\left(t_{j}-t_{j-1}\right)\right\} \\
& \cdot f\left(x^{(0)}\right)\left(\prod_{l=1}^{n}(2 \pi)^{-d} d p^{(l-1)} d x^{(l-1)}\right) d x^{(n)},
\end{aligned}
$$

where $t_{j}=j t / n$, and $p^{(j)}=P\left(t_{j}\right), x^{(j)}=X\left(t_{j}\right), j=0,1, \ldots, n$. The integrand of the integral on the right of $(6.5)$ is rewritten as

$$
\begin{gathered}
\overline{g\left(x^{(n)}\right)} \prod_{j=1}^{n}\left\{\exp \left[i x^{(j)} \cdot p^{(j-1)}\right] \exp \left[-(t / n)\left(c^{2}\left(p^{(j-1)}\right)^{2}+m^{2} c^{4}\right)^{1 / 2}\right]\right. \\
\left.\cdot \exp \left[-i x^{(j-1)} \cdot p^{(j-1)}\right]\right\} \exp \left\{i \sum_{k=1}^{n}(e / c) A\left(\frac{x^{(k-1)}+x^{(k)}}{2}\right)\right. \\
\left.\cdot\left(x^{(k)}-x^{(k-1)}\right)-e \Phi\left(\frac{x^{(k-1)}+x^{(k)}}{2}\right)(t / n)\right\} f\left(x^{(0)}\right) .
\end{gathered}
$$

Now multiply it by $\exp \left[m c^{2} t\right]$ and integrate it with respect to all the $p^{(j)}$. Recall $k_{0}(t, x)$ is the fundamental solution of the Cauchy problem for (4.1). Then we have

$$
\begin{aligned}
\left(e^{-t\left[H-m c^{2}\right]} g, f\right)= & \lim _{n \rightarrow \infty} \int_{\mathbb{R}^{d}}^{n+1} \cdots \int_{\mathbb{R}^{d}}^{+1} \overline{g\left(x^{(n)}\right)} k_{0}\left(t / n, x^{(0)}-x^{(1)}\right) \cdots \cdot k_{0}\left(t / n, x^{(n-1)}-x^{(n)}\right) \\
& \cdot \exp \left[-S_{n}^{*}\left(x^{(0)}, \ldots, x^{(n)}\right)\right] f\left(x^{(0)}\right) d x^{(0)} d x^{(1)} \cdots d x^{(n)}
\end{aligned}
$$


where $S_{n}^{*}$ is the complex conjugate of $S_{n}$ in (4.7). Since $f$ is arbitrary and the kernel $k_{0}(t, x)$ is nonnegative, it follows that

$$
\begin{aligned}
\left(e^{-t\left[H-m c^{2}\right]} g\right)(x)= & \lim _{n \rightarrow \infty} \overbrace{\int_{\mathbb{R}^{d}}}^{n} \int_{\mathbb{R}^{d}}^{n} k_{0}\left(t / n, x^{(0)}-x^{(1)}\right) \cdots k_{0}\left(t / n, x^{(n-1)}-x^{(n)}\right) \\
& \cdot \exp \left[-S_{n}\left(x^{(0)}, \ldots, x^{(n)}\right)\right] g\left(x^{(n)}\right) d x^{(1)} \ldots \cdot d x^{(n)},
\end{aligned}
$$

The integral on the right of (6.7) is the same as that of (4.6), and we have seen in Sect. 4 that its limit has the path integral representation (2.1) with (2.2).

Acknowledgements. One of us (T. I.) is indebted to Professors H. Araki, M. Fukushima, S. Kotani and Y. Okabe for useful informative discussions on some physical aspects and the Lévy process at an early stage of the present work. He is also grateful to Professor S. R. S. Varadhan for stimulating discussions at Les Houches Summer School in Theoretical Physics 1984. The other (H. T.) would like to thank Professor A. Kanazawa for his warm encouragement. Last but not least we are grateful to Professor J. R. Klauder for useful informative discussions and his unceasing encouragement.

The referee is thanked for a useful comment on the expression (2.2).

\section{References}

1. Berezin, F.A., Subin, M. A.: Symbols of operators and quantization. Colloq. Math. Soc. Janos Bolyai 5. Hilbert Space Operators, 21-52, Tihany 1970

2. Calderón, A. P., Vaillancourt, R.: A class of bounded pseudo-differential operators. Proc. Nat. Acad. Sci. USA 69, 1185-1187 (1972)

3. Daubechies, I., Lieb, E.H.: one-electron relativistic molecules with Coulomb interaction. Commun. Math. Phys. 90, 497-510 (1983)

4. Daubechies, I.: One electron molecules with relativistic kinetic energy: Properties of the discrete spectrum. Commun. Math. Phys. 94, 523-535 (1984)

5. Erdélyi, A.: Higher transcendental functions, Vol. 2. New York: McGraw-Hill 1953

6. Erdélyi, A.: Tables of integral transforms, Vol. 2, New York: McGraw-Hill 1954

7. Garrod, C.: Hamiltonian path-integral methods. Rev. Mod. Phys. 38, 483-494 (1966)

8. Gaveau, B.: Representation formulas of the Cauchy problem for hyperbolic systems generalizing Dirac system. J. Funct. Anal. 58, 310-319 (1984)

9. Gaveau, B., Jacobson, T., Kac, M., Schulman, L. S.: Relativistic extension of the analogy between quantum mechanics and Brownian motion. Phys. Rev. Lett. 53 (5), 419-422 (1984)

10. Grossmann, A., Loupias, G., Stein, E. M.: An algebra of pseudodifferential operators and quantum mechanics in phase space. Ann. Inst. Fourier 18, 343-368 (1968)

11. Herbst, I. W.: Spectral theory of the operator $\left(p^{2}+m^{2}\right)^{1 / 2}-Z e^{2} / r$. Commun. Math. Phys. 53, 285294 (1977)

12. Hörmander, L.: The analysis of linear partial differential operators III. Berlin, Heidelberg, New York, Tokyo: 1985

13. Ichinose, T., Tamura, H.: Propagation of a Dirac particle. A path integral approach. J. Math. Phys. 25, 1810-1819 (1984)

14. Ichinose, T.: Path integral for a hyperbolic system of the first order. Duke Math. J. 51, 1-36 (1984)

15. Ichinose, T.: Path integral formulation of the propagator for a two-dimensional Dirac particle. Physica 124 A, 419-426 (1984)

16. Ikeda N., Watanabe, S.: Stochastic differential equations and diffusion processes. Amsterdam, Tokyo: North-Holland/Kodansha 1981

17. Itô, K.: Stochastic processes. Lecture Notes Series, 16, Aårhus University 1969

18. Kumano-go, H.: Pseudo-differential operators of multiple symbol and the Calderón-Vaillancourt theorem. J. Math. Soc. Jpn 27, 113-120 (1975)

19. Kumano-go, H.: Pseudo-differential operators. Cambridge, Massachusetts: The MIT Press 1981 
20. Landau, L. D., Lifschitz, E. M.: Course of theoretical physics, Vol. 2. The classical theory of fields, 4th revised English ed. Oxford: Pergamon Press 1975

21. Mizrahi, M. M.: The Weyl correspondence and path integrals. J. Math. Phys. 16, 2201-2206 (1975)

22. Mizrahi, M. M.: Phase space path integrals, without limiting procedure. J. Math. Phys. 19, 298-307 (1978); Erratum, ibid. 21, 1965 (1980)

23. Muramatu, T., Nagase, M.: On sufficient conditions for the boundedness of pseudo-differential operators. Proc. Jpn Acad. 55 (A), 293-296 (1979)

24. Nagase, M.: The $L^{p}$-boundedness of pseudo-differential operators with non-regular symbols. Commun. Partial. Differ. Equations., 2, 1045-1061 (1977)

25. Reed, M., Simon, B.: Methods of modern mathematical physics, IV: Analysis of operators. New York: Academic Press 1978

26. Shubin, M. A.: Essential self-adjointness of uniformly hypoelliptic operators. Vestn. Mosk. Univ. Ser. I. 30, 91-94 (1975); English transl. Mosc. Univ. Math. Bull. 30, 147-150 (19j5)

27. Simon, B.: Functional integration and quantum physics. New York: Academic Press 1979

Communicated by H. Araki

Received August 19, 1985; in revised form January 14, 1986 
